4. Majumder S, et al. Life-long rapamycin administration ameliorates age-dependent cognitive deficits by reducing IL-1 $\beta$ and NMDA signaling. Aging Cell. 2012;11(2):326-335.

5. Caccamo A, Majumder S, Richardson A, Strong $\mathrm{R}$, Oddo S. Molecular interplay between $\mathrm{mTOR}$, amyloid-beta, and tau: Effects on cognitive impairments. J Biol Chem. 2010;285(17):13107-13120

6. Spilman $S$, et al. Inhibition of mTOR by rapamycin abolishes cognitive deficits and reduces amyloid- $\beta$ levels in a mouse model of Alzheimer's disease. PLoS One. 2010;5(4):e9979.

7. Majumder S, Richardson A, Strong R, Oddo S. Inducing autophagy by rapamycin before but not after plaques and tangles formation ameliorates cognitive deficits in a mouse model of Alzheimer's disease. PLoS One. 2011;6(9):e25416.

8. Ramos FJ, et al. Rapamycin reverses elevated mTORC1 signaling in lamin A/C-deficient mice, rescues cardiac and skeletal muscle function, and extends survival. Sci Transl Med. 2012;4(144):144ra103.

9. Zhang Y, et al. Rapamycin extends life and health in C57BL/ 6 mice [published online ahead of print May 16, 2013]. J Gerontol A Biol Sci Med Sci. doi:10.1093/gerona/glt056.

10. Ravikumar B, et al. Inhibition of mTOR induces autophagy and reduces toxicity of polyglutamine expansions in fly and mouse models of Huntington disease. Nat Genet. 2004;36(6):585-595.

11. Wilkinson JE, et al. Rapamycin slows aging in mice. Aging Cell. 2012;11(4):675-682.

12. Ikeno Y, Lew CM, Cortez LA, Webb CR, Lee S, Hubbard GB. Do long-lived mutant and calorierestricted mice share common anti-aging mechanisms? - a pathological point of view. Age (Dordr). 2006;28(2):163-171.

13. Miller RA, et al. Rapamycin, but not resveratrol or simvastatin, extends lifespan of genetically heterogeneous mice. J Gerontol A Biol Sci Med Sci. 2011;66(2):191-20.

14. Fujishita T, Aoki K, Lane HA, Aoki M, Taketo MM. Inhibition of the mTORC1 pathway suppresses intestinal polyp formation and reduces mortality in ApcDelta716 mice. Proc Natl Acad Sci U S A. 2008;105(36):13544-13549.

15. Comas M, et al. New nanoformulation of rapamycin Rapatar extends lifespan in homozygous $p 53^{--}$ mice by delaying carcinogenesis. Aging (Albany NY). 2012;4(10):715-722.

16. Anisimov VN, et al. Rapamycin extends maximal lifespan in cancer-prone mice. Am J Pathol. 2010;175(5):2092-2097.
17. Livi CB, et al. Rapamycin extends life span of $\mathrm{Rb} 1^{+/}$ mice by inhibiting neuroendocrine tumors. Aging (Albany NY). 2013;5(2):100-110.

18. Richardson A, McCarter R. Mechanism of food restriction: change of rate or change of set point? In: Ingram DK, Baker GT, Shock NW, eds. The Potential for Nutritional Modulation of Aging Processes. Trumbull, Connecticut, USA: Food and Nutrition Press, Inc.; 1991:177-192.

19. Mueller MA, Beutner F, Teupser D, Ceglarek U, Thiery J. Prevention of atherosclerosis by the mTOR inhibitor everolimus in $\mathrm{LDLR}^{-/-}$mice despite severe hypercholesterolemia. Atherosclerosis. 2008;198(1):39-48.

20. Cohen EEW. mTOR: The mammalian target of replication. J Clin Oncol. 2008;26(3):348-349.

21. Scott A, Karnad S, Karnad A, Brenner AJ. Common toxicities of mammalian target of rapamycin inhibitors. Target Oncol. 2011;6(2):125-129.

22. Hinojosa CA, et al. Enteric-delivered rapamycin enhances resistance of aged mice to pneumococcal pneumonia through reduced cellular senescence. Exp Gerontol. 2012;47(12):958-965.

23. Mita M, Mita A. Are we ready to move away from nature? The rapamycin story. Target Oncol. 2011;6(2):63-64.

\title{
The future of molecular chaperones and beyond
}

\author{
Rona G. Giffard, ${ }^{1}$ Alberto J.L. Macario, ${ }^{2,3}$ and Everly Conway de Macario²
}

\begin{abstract}
1Department of Anesthesia, Stanford University School of Medicine, Stanford, California, USA. 2Department of Microbiology and Immunology, School of Medicine, University of Maryland at Baltimore, and IMET, Columbus Center, Baltimore, Maryland, USA.
\end{abstract} ${ }^{3}$ Istituto EuroMediterraneo di Scienza e Tecnologia (IEMEST), Palermo, Italy.

\begin{abstract}
Protection of hair cells by HSP70 released by supporting cells is reported by May et al. in this issue of the JCI. Their findings suggest a new way to reduce ototoxicity from therapeutic medications and raise larger questions about the role and integration of heat shock proteins in non-cell-autonomous responses to stress. Increasing evidence suggests an important role for extracellular heat shock proteins in both the nervous system and the immune system. The work also suggests that defective chaperones could cause ear disease and supports the potential use of chaperone therapeutics.
\end{abstract}

\section{Heat shock proteins}

Heat shock proteins (HSPs) are the product of heat shock genes, initially defined as genes induced by a short, sudden temperature elevation above normal; however, many HSP genes are induced by stressors other than heat shock, and some are constitutively expressed essential proteins. Initially, HSP70 was recognized as an intracellular chaperone with a central role in protein folding of nascent and denatured proteins (1-4), and extracellular HSP70 was thought to be the result of cell death. A body of work has now demonstrated that HSP70 is released from a wide

Conflict of interest: The authors have declared that no conflict of interest exists.

Citation for this article: J Clin Invest. 2013; 123(8):3206-3208. doi:10.1172/JCI70799. variety of cells via a noncanonical secretion pathway $(5,6)$, as the sequence is devoid of a consensus secretory signal, and inhibitors of the common secretory pathway fail to block secretion. HSP70 is thought to share secretion pathways used by other leaderless proteins that are nevertheless secreted, such as IL- $1 \beta$, but this remains an area of active research.

HSP70 is a highly evolutionarily conserved molecule, from bacteria to people. In humans, it has expanded from the single DNAK protein found in bacteria to a family of at least 17 members in humans (7). Further, some of the genes likely produce more than one mRNA and protein variant. Because it is such an ancient protein and because it has always been involved with stress sensing and stress resistance, it is not surprising that it also participates with two organ systems that have evolved to sense environmental challenges and respond to them, the nervous system and the immune system. Indeed, it is especially in these two systems that HSP70 roles beyond the classical intracellular locations have begun to be revealed.

HSP70 has been shown to have many paracrine effects, especially in the setting of injury and repair. Extracellular HSP70, both free and endosome/vesicle-associated, provides information about stress and cell injury. It acts as an alarmin or damageassociated molecular pattern; activates signaling cascades, including the cytokineinducing MyD88/IRAK/NF-кB pathway (8); regulates signaling pathways, including FOXO and the MAPK p38 (9); and acts on numerous cell types, including immune cells, epithelial cells, hepatocytes, and neurons. For example, HSP70 plays a central role in the regulation of inflammation and regeneration following muscle injury (9).

The article by May et al. reports that HSP70 release from supporting cells is necessary and sufficient to protect mechanosensory hair cell neurons from the toxic effects of aminoglycoside antibiotics (10). This is of broad importance, as there are 
currently no known ways to reduce the ototoxicity of aminoglycoside antibiotics, which are widely used and cause significant hearing loss or impaired balance in up to $20 \%$ of patients receiving these drugs. It is estimated that 500,000 patients each year in the United States suffer hearing loss or balance impairment from the use of ototoxic therapeutic medications.

A unique organotypic utricle culture system was used to investigate the mechanism of HSP70 protection in this setting, and the authors demonstrate that the release of HSP70 from the glia-like supporting cells that nestle at the base of the hair cells leads to this protection. There are two central findings here that advance understanding in this field: first, that the neighboring glial cells release the HSP70 that provides protection; and second, that extracellular HSP70 appears sufficient to protect the neurons, without requiring significant uptake. This points to a critical role for signaling by HSP70, rather than its canonical function as a direct molecular chaperone. The work extends the broader understanding of the role of extracellular HSP70 as a molecule conveying signals from one cell to another, likely via binding to cell surface receptors; however, the receptor for HSP70 in this setting is unknown.

In addition to being found in plasma in the setting of disease, it is now clear that stresses that do not induce substantial cell death, including psychological stress and exercise, lead to significant increases in extracellular HSP70 (5). The findings reported by May et al. (10) also suggest the possibility that defective chaperone secretion or function could directly contribute to hearing loss/balance disturbance if the hair cell is not protected, similar to what happens in other conditions in which defective chaperones contribute to pathogenesis (11).

Immune and nervous system reflexes Both functional and anatomic connections between the immune system and the CNS are now recognized. There are immune neuronal reflexes that allow communication of immune system information from the periphery to the CNS via sensory afferents traveling through the vagus nerve, as well as neuronal efferents from the nucleus ambiguous and dorsal motor nucleus, again traveling through the vagus nerve to innervate immune organs, including the spleen, lymph nodes, and thymus. In addition to signaling via sensory neurons, the peripheral immune system can communicate with the brain via soluble mediators (for review see ref. 12). This understanding has been in part a result of the recognition that neurons express receptors for immune mediators, and immune cells express receptors for neurotransmitters. This raises the question of whether HSP70 signals to hair cell neurons via one of the known or suggested HSP70 receptors discussed below, most of which have known immune system functions.

\section{HSP70 and the nervous system}

HSPs have been shown to play a role in neuronal survival and communication between glial cells and neurons. The report by May et al. (10) is consistent with a growing body of literature suggesting that HSP70 can be provided to neurons by glial cells (13). This is especially beneficial since neurons themselves have a reduced ability to induce HSP70 in response to stress, reflecting differences in transcription factor expression and regulation (14). Early work suggested that HSP70 could be transferred to axons from neighboring glia, and extracellular HSP70 was shown to protect motor neurons $(13,15)$. HSPs have been shown to protect the nervous system from a variety of stresses and neurodegenerative diseases $(16,17)$. Further interesting work in the nematode C. elegans describes a different interaction between the induction of HSP70 and the nervous system, allowing the intact organism to distinguish acute stress from chronic stress. Heat-sensing neurons inhibited induction of the heat shock response with chronic stress, demonstrating noncell-autonomous control of HSP induction (reviewed in ref. 18). The work by May et al. extends current knowledge of the roles of extracellular HSP70 in the CNS (10).

\section{HSP70 and the immune system}

The article by May et al. (10) raises an important question: which receptors transduce the protective signal carried by HSP70? This merits further investigation because the answer may indicate ways to improve, stimulate, or simulate the interaction of HSP70 with its surface receptor for therapeutic protective purposes. There are already clues in the literature pointing to a variety of possible receptors, some thought to mediate internalization and others thought to mediate signaling, but our understanding of this area is still in an early stage.

Much of the work to better understand the role of extracellular HSP70 has been performed in the immune system. As is often the case, the picture that is developing is complex. HSP70 has been shown to be both strongly immunostimulatory and immunosuppressive, depending on the cell type and context. Much of this work has focused on the ability of HSP70 to modify the response to tumors, facilitating the elimination of tumor cells, but there are also intriguing data in the setting of chronic inflammatory disease, including rheumatoid arthritis, diabetes, and atherosclerosis, in which HSP70 may exert immune modulatory antiinflammatory effects via $T$ regulatory lymphocytes $(19,20)$. HSP70 can induce proinflammatory cytokine production in a calciumand NF-кB-dependent manner via TLR2 and 4 (8), as well as inhibit inflammatory cytokine production by inhibiting NF-кB activation (17).

Most of the potential HSP70 receptors identified thus far are best known for their function in the immune system, though they are also expressed on other cell types. Possible receptors include members of the Toll-like receptor family, especially TLR2 and 4, as well as some scavenging receptors, including LOX-1, SREC-1, and FEEL1, representing another family of pattern recognition receptors and C-type lectins (21). Also, the $\alpha 2$-macroglobulin receptor CD91 (22) was implicated in allowing highly efficient antigen presentation by the complex of antigenic peptide with HSP70, which requires APCs. Thus, extracellular HSP70 participates in both the innate immune response and the adaptive immune response.

A growing body of work documents many roles for extracellular HSP70, especially in immune regulation, but also increasingly in communication between cells and between tissues and the immune system. Key questions remain with regard to the receptors for HSP70 on different cells, including neurons, as well as to the signaling pathways involved and how this signaling alters the cell's phenotype. The role of extracellular HSP70 in many disease states and in aging are also important outstanding questions. The possibilities of using HSPs for neuroprotection are tantalizing but will require greater understanding for successful translation into clinical use.

\section{Acknowledgments}

Rona G. Giffard is supported in part by NIH grants (NS053898, GM049831, and NS084396) and the Department of Anesthesia at Stanford University. Alberto J.L. Macario is supported by the IEMEST. 
Address correspondence to: Rona G. Giffard, Stanford University School of Medicine, 300 Pasteur Drive, Grant Building S272A, Stanford, California 94305-5117, USA. Phone: 650.725.8482; Fax: 650.725.8052; E-mail: rona.giffard@stanford.edu.

1. Georgopoulos C, Welch WJ. Role of the major heat shock proteins as molecular chaperones. Ann Rev Cell Biol. 1993;9:601-634.

2. Lindquist S, Craig EA. The heat-shock proteins. Ann Rev Genet. 1988;22:631-677.

3. Mayer MP, Bukau B. Hsp70 chaperones: cellular functions and molecular mechanism. Cell Mol Life Sci. 2005;62(6):670-684.

4. Young JC, Agashe VR, Siegers K, Hartl FU. Pathways of chaperone-mediated protein folding in the cytosol. Nat Rev Mol Cell Biol. 2004;5(10):781-791.

5. Fleshner M, Johnson JD. Endogenous extra-cellular heat shock protein 72 : releasing signal(s) and function. Int J Hypertherm. 2005;21(5):457-471.

6. Mambula SS, Calderwood SK. Heat shock protein 70 is secreted from tumor cells by a nonclassical pathway involving lysosomal endosomes. J Immunol. 2006;177(11):7849-7857.

7. Brocchieri L, Conway de Macario E, Macario AJL. hsp70 genes in the human genome: conservation and differentiation patterns predict a wide array of overlapping and specialized functions. BMC Evol Biol. 2008;8:19.

8. Asea A, et al. Novel signal transduction pathway utilized by extracellular HSP70: role of toll-like receptor (TLR) 2 and TLR4.J Biol Chem. 2002;277(17):15028-15034.

9. Senf SM, Howard TM, Ahn B, Ferreira LF, Judge AR. Loss of the inducible hsp70 delays the inflammatory response to skeletal muscle injury and severely impairs muscle regeneration. PLoS One. 2013;8(4):e62687.

10. May LA, et al. Inner ear supporting cells protect hair cells by secreting HSP70. J Clin Invest. 2013;123(8):3577-3587.

11. Macario AJL, Conway de Macario E. Sick chaperones, cellular stress, and disease. $N$ Engl J Med. 2005;353(14):1489-1501.

12. Andersson U, Tracey KJ. Neural reflexes in inflammation and immunity. $J$ Exp Med. 2012;209(6):1057-1068

13. Tytell M. Release of heat shock proteins (Hsps) and the effects of extracellular Hsps on neural cells and tissues. Int J Hypertherm. 2005;21(5):445-455.

14. Tonkiss J, Calderwood SK. Regulation of heat shock gene transcription in neuronal cells. Int J Hypertherm. 2005;21(5):433-444.

15. Guzhova I, et al. In vitro studies show that Hsp70 can be released by glia and that exogenous Hsp70 can enhance neuronal stress tolerance. Brain Res. 2001;914(1-2):66-73

16. Brown IR. Heat shock proteins and protection of the nervous system. Ann N Y Acad Sci. 2007;1113:147-158

17. Giffard RG, Han R-Q, Emery JF, Duan M, Pittet JF. Regulation of apoptotic and inflammatory cell signaling in cerebral ischemia: the complex roles of heat shock protein 70 . Anesthesiology. 2008;109(2):339-348.

18. Prahlad V, Morimoto RI. Integrating the stress response: lessons for neurodegenerative diseases from C. elegans. Trends Cell Biol. 2009;19(2):52-61.

19. Pockley AG, Calderwood S, Multhoff G. The atheroprotective properties of Hsp70: a role for Hsp70-endothelial interactions? Cell Stress Chap. 2009;14(6):545-553.

20. Van Eden W, Wick G, Albani S, Cohen I. Stress, heat shock proteins, and autoimmunity. Ann NY Acad Sci. 2007;1113:217-237.

21. Thériault JR, Mambula SS, Sawamura T, Stevenson MA, Calderwood SK. Extracellular HSP70 binding to surface receptors present on antigen presenting cells and endothelial/epithelial cells. FEBS Lett. 2005;579(9):1951-1960.

22. Basu S, Binder RJ, Ramalingam T, Srivastava PK. CD91 is a common receptor for heat shock proteins gp96, hsp90, hsp70, and calreticulin. Immunity. 2001;14(3):303-313.

\title{
Chronic THC intake modifies fundamental cerebellar functions
}

\author{
Nephi Stella \\ Department of Pharmacology, University of Washington, Seattle, Washington, USA.
}

\begin{abstract}
Delta9-tetrahydrocannabinol (THC), the principal bioactive component in the Cannabis plant, is truly a captivating drug. Acute and chronic THC intake produces a spectrum of biological effects ranging from transient psychotropic effects to prolonged medicinal benefits, many of which have been fostered for centuries by our society. In the July 2013 issue of the JCI, Cutando et al. combined mouse genetics with classic mouse behavioral analysis to deepen our understanding of the physiological consequence of subchronic THC intake on eyeblink reflexes, a fundamental neuronal adaptive response, revealing that this regimen leads to downregulation of the cannabinoid $\mathrm{CB}_{1}$ receptor (referred to as $\mathrm{CB} 1$ in the Cutando et al. article) in cerebellar stress fibers and the activation of microglia, raising provocative new questions about the safety profile of regimented THC intake.
\end{abstract}

\section{Consequences in the blink of an eye}

Our detailed understanding of the molecular mechanism of action of THC on neuronal activity began 20 years ago with the molecular identification of the gene encoding for $\mathrm{CB}_{1}$ receptors, which mediate the psychoactive effects produced by

Conflict of interest: The author has declared that no conflict of interest exists.

Citation for this article: J Clin Invest. 2013; 123(8):3208-3210. doi:10.1172/JCI70226. this compound (1). $\mathrm{CB}_{1}$ receptors represent one of the most abundant $G$ proteincoupled receptors expressed in the brain, although the expression level and coupling mechanism vary according to neuron type (2). Activation of $\mathrm{CB}_{1}$ receptors by endogenous cannabinoid ligands, the endocannabinoids (eCBs), modulates neuronal activity at 4 overarching levels: by guiding neuronal patterning and connectivity during brain development (3); by modulating neurotransmitter release probability (4); by mediating short-term and long-term synaptic plasticity and shaping neuronal network connectivity $(5,6)$; and by controlling the expression of prosurvival proteins that promote neuronal endurance in response to brain injuries and pathologies (7). Unlike eCBs, THC activates $\mathrm{CB}_{1}$ receptors with its own pharmacodynamic profile and thus may impinge upon neuronal activity at these 4 levels (8).

The effect of THC on higher sensory, intentional, and memory processes has been intensely studied, but more recently, a major research focus has been on how THC affects neuronal activity at the systems level, revealing the breadth of THC's impact on behavior. In the current study, Cutando et al. (9) investigated the effect of subchronic THC exposure on cerebellar function. The cerebellum represents a key relay structure involved in the fine tuning of motor coordination, the implementation of associative learning, and the processing of temporal operations. Cutando et al. (9) found that subchronic THC exposure led to the impair- 